\title{
Heterogeneity between primary colon carcinoma and paired lymphatic and hepatic metastases
}

\author{
HUANRONG LAN ${ }^{1}$, KETAO JIN $^{2,3}$, BOJIAN XIE $^{4}$, NA HAN $^{5}$, BINBIN CUI $^{2}$, FEILIN CAO $^{2}$ and LISONG TENG \\ Departments of ${ }^{1}$ Gynecology and Obstetrics, and ${ }^{2}$ Surgical Oncology, Taizhou Hospital, Wenzhou Medical College, \\ Linhai, Zhejiang; ${ }^{3}$ Department of Surgical Oncology, First Affiliated Hospital, College of Medicine, Zhejiang University, \\ Hangzhou, Zhejiang; ${ }^{4}$ Department of Surgical Oncology, Sir Run Run Shaw Hospital, College of Medicine, \\ Zhejiang University, Hangzhou, Zhejiang; ${ }^{5}$ Cancer Chemotherapy Center, Zhejiang Cancer Hospital, \\ Zhejiang University of Chinese Medicine, Hangzhou, Zhejiang, P.R. China
}

Received January 26, 2012; Accepted May 8, 2012

DOI: $10.3892 / \mathrm{mmr} .2012 .1051$

\begin{abstract}
Heterogeneity is one of the recognized characteristics of human tumors, and occurs on multiple levels in a wide range of tumors. A number of studies have focused on the heterogeneity found in primary tumors and related metastases with the consideration that the evaluation of metastatic rather than primary sites could be of clinical relevance. Numerous studies have demonstrated particularly high rates of heterogeneity between primary colorectal tumors and their paired lymphatic and hepatic metastases. It has also been proposed that the heterogeneity between primary colon carcinomas and their paired lymphatic and hepatic metastases may result in different responses to anticancer therapies. The heterogeneity in primary colon carcinoma and corresponding metastases by genome-wide gene expression analysis has not been extensively studied. In the present study, we investigated the differentially expressed genes between a primary colon carcinoma specimen (obtained from a 40-year-old female colon carcinoma patient with lymphatic and hepatic metastases) and its paired lymphatic and hepatic metastases by genome-wide gene expression analysis using GeneChip HGU133Plus2.0 expression arrays. Our results demonstrate that genome-wide gene expression varies between primary colon carcinoma and its paired lymphatic and hepatic metastases.
\end{abstract}

Correspondence to: Dr Lisong Teng, Department of Surgical Oncology, First Affiliated Hospital, College of Medicine, Zhejiang University, 79, Qingchun Road, Hangzhou, Zhejiang 310003, P.R. China

E-mail: jinketao2001@zju.edu.cn

Dr Feilin Cao, Department of Surgical Oncology, Taizhou Hospital, Wenzhou Medical College, 150 Ximen Road, Linhai, Zhejiang 317000, P.R. China

E-mail:dr.caofeilin@yahoo.com.cn

Key words: colon carcinoma, hepatic metastasis, heterogeneity, genome-wide gene expression, functional gene

\section{Introduction}

Intratumor heterogeneity is one of the recognized characteristics of human tumors, which occurs on multiple levels, including genetic, protein and macroscopic, in a wide range of tumors, including breast, colorectal cancer (CRC), nonsmall cell lung cancer (NSCLC), prostate, ovarian, pancreatic, gastric, brain and renal clear cell carcinoma (1). Over the past decade, a number of studies have focused on the heterogeneity found in primary tumors and related metastases with the consideration that the evaluation of metastatic rather than primary sites may be of clinical relevance. Numerous reports have evaluated the genetic heterogeneity in primary tumors and corresponding metastases in a range of solid tumors, including breast cancer (2-9), CRC (10-13) and NSCLC (14,15).

In $\mathrm{CRC}$, the heterogeneity of the epidermal growth factor receptor $(E G F R)$ gene status and its downstream signalling proteins, including $K R A S, B R A F$ and PIK3CA mutations, have been identified by comparing primary tumors with corresponding metastases, respectively (10-13). Baldus et al demonstrated particularly high rates of heterogeneity between primary colorectal tumors and lymph node metastases (10). Molinari et al analyzed EGFR gene status and protein expression, KRAS/BRAF mutations and PTEN expression in primary tumors and metastases in 38 metastatic CRC patients, and found $E G F R$ gene deregulation in $69.4 \%$ of primary tumors and $80.6 \%$ of metastases, and KRAS mutations in $43.2 \%$ of primary tumors and $40.5 \%$ of metastases (11). However, the heterogeneity in primary CRC and corresponding metastases at the genome-wide gene expression level has not been extensively investigated.

Therefore, in the present study, we investigated the heterogeneity in primary colon carcinoma tissue and its corresponding lymphatic and hepatic metastatic tissues obtained from a female metastatic colon cancer patient, focusing on genome-wide gene expression.

\section{Patients and methods}

Patient and tissue samples. Tumor specimens were obtained at initial surgery from a 40 -year-old female colon carcinoma 
Table I. Genes differentially expressed in the primary colon carcinoma specimen and its paired lymphatic metastasis.

\begin{tabular}{|c|c|c|c|c|}
\hline Probe set ID & Fold change & Gene symbol & Entrez gene & Gene title \\
\hline 201847_at & 23.27895 & LIPA & 3988 & Lipase A, lysosomal acid, cholesterol esterase \\
\hline 203323_at & 20.588184 & CAV2 & 858 & Caveolin 2 \\
\hline 203324_s_at & 25.194075 & CAV2 & 858 & Caveolin 2 \\
\hline 203571_s_at & 23.1392 & $\mathrm{C} 10$ or $\mathrm{f} 116$ & 10974 & Chromosome 10 open reading frame 116 \\
\hline 203639_s_at & 69.52935 & FGFR2 & 2263 & Fibroblast growth factor receptor 2 \\
\hline 203697_at & 20.352211 & FRZB & 2487 & Frizzled-related protein \\
\hline 204010_s_at & 34.406235 & KRAS & 3845 & v-Ki-ras2 Kirsten rat sarcoma viral oncogene homolog \\
\hline 204035_at & 39.844757 & SCG2 & 7857 & Secretogranin II (chromogranin C) \\
\hline 204439_at & 46.355186 & IFI44L & 10964 & Interferon-induced protein 44-like \\
\hline 204802_at & 113.641525 & RRAD & 6236 & Ras-related associated with diabetes \\
\hline 204803_s_at & 41.770977 & RRAD & 6236 & Ras-related associated with diabetes \\
\hline 205151_s_at & 36.290764 & KIAA0644 & 9865 & KIAA0644 gene product \\
\hline 205625_s_at & 29.481396 & CALB1 & 793 & Calbindin $1,28 \mathrm{kDa}$ \\
\hline 205696_s_at & 45.68169 & GFRA1 & 2674 & GDNF family receptor $\alpha 1$ \\
\hline 205778_at & 28.692463 & KLK7 & 5650 & Kallikrein-related peptidase 7 \\
\hline 205818_at & 109.04431 & $\mathrm{DBC} 1$ & 1620 & Deleted in bladder cancer 1 \\
\hline 205830_at & 20.343859 & CLGN & 1047 & Calmegin \\
\hline 206091_at & 25.613195 & MATN3 & 4148 & Matrilin 3 \\
\hline 206340_at & 24.728613 & NR1H4 & 9971 & Nuclear receptor subfamily 1 , group $\mathrm{H}$, member 4 \\
\hline 206525_at & 25.197699 & GABRR1 & 2569 & $\gamma$-aminobutyric acid (GABA) receptor, rho 1 \\
\hline 206619_at & 34.690308 & DKK4 & 27121 & Dickkopf homolog 4 (Xenopus laevis) \\
\hline 206877_at & 20.351955 & MXD1 & 4084 & MAX dimerization protein 1 \\
\hline 207254_at & 48.329205 & SLC15A1 & 6564 & $\begin{array}{l}\text { Solute carrier family } 15 \text { (oligopeptide transporter), } \\
\text { member } 1\end{array}$ \\
\hline 207509_s_at & 51.56186 & LAIR2 & 3904 & Leukocyte-associated immunoglobulin-like receptor 2 \\
\hline 207583_at & 40.26811 & $\mathrm{ABCD} 2$ & 225 & ATP-binding cassette, sub-family D (ALD), member 2 \\
\hline 207610_s_at & 23.911833 & EMR2 & 30817 & $\begin{array}{l}\text { Egf-like module containing, mucin-like, hormone } \\
\text { receptor-like } 2\end{array}$ \\
\hline 208146_s_at & 89.68937 & CPVL & 54504 & Carboxypeptidase, vitellogenic-like \\
\hline 208261_x_at & 29.10015 & IFNA10 & 3446 & Interferon, $\alpha 10$ \\
\hline 208481_at & 26.099915 & ASB4 & 51666 & Ankyrin repeat and SOCS box-containing 4 \\
\hline 208791_at & 46.167988 & CLU & 1191 & Clusterin \\
\hline 209406_at & 29.201277 & BAG2 & 9532 & BCL2-associated athanogene 2 \\
\hline 209792_s_at & 38.65837 & KLK10 & 5655 & Kallikrein-related peptidase 10 \\
\hline 209821_at & 205.30872 & IL33 & 90865 & Interleukin 33 \\
\hline 210510_s_at & 27.80813 & NRP1 & 8829 & Neuropilin 1 \\
\hline 210931_at & 24.963585 & RNF6 & 6049 & Ring finger protein $(\mathrm{C} 3 \mathrm{H} 2 \mathrm{C} 3$ type $) 6$ \\
\hline 211161_s_at & 30.307695 & COL3A1 & 1281 & Collagen, type III, $\alpha 1$ \\
\hline 211401_s_at & 20.871784 & FGFR2 & 2263 & Fibroblast growth factor receptor 2 \\
\hline 213238_at & 42.231457 & ATP10D & 57205 & ATPase, class V, type 10D \\
\hline 213428_s_at & 44.14465 & COL6A1 & 1291 & Collagen, type VI, $\alpha 1$ \\
\hline 213432_at & 189.0725 & MUC5B & 727897 & Mucin 5B, oligomeric mucus/gel-forming \\
\hline 213874_at & 60.066086 & SERPINA4 & 5267 & $\begin{array}{l}\text { Serpin peptidase inhibitor, clade A ( } \alpha-1 \text { antiproteinase, } \\
\text { antitrypsin), member } 4\end{array}$ \\
\hline 214044_at & 23.23853 & RYR2 & 6262 & Ryanodine receptor 2 (cardiac) \\
\hline 214121_x_at & 28.547356 & PDLIM7 & 9260 & PDZ and LIM domain 7 (enigma) \\
\hline 214146_s_at & 40.065388 & PPBP & 5473 & $\begin{array}{l}\text { Pro-platelet basic protein [chemokine (C-X-C motif) } \\
\text { ligand 7] }\end{array}$ \\
\hline 214453_s_at & 33.425632 & IFI44 & 10561 & Interferon-induced protein 44 \\
\hline \multirow[t]{2}{*}{ 214567_s_at } & 25.704966 & XCL1 & 6375 & Chemokine (C motif) ligand 1 \\
\hline & & XCL2 & 6846 & Chemokine (C motif) ligand 2 \\
\hline 214769_at & 31.164827 & CLCN4 & 1183 & Chloride channel 4 \\
\hline 214811_at & 62.246216 & RIMBP2 & 23504 & RIMS binding protein 2 \\
\hline 214823_at & 36.446995 & ZNF204 & 7754 & Zinc finger protein 204 pseudogene \\
\hline 214952_at & 31.43681 & NCAM1 & 4684 & Neural cell adhesion molecule 1 \\
\hline 215081_at & 38.364174 & KIAA1024 & 23251 & KIAA1024 \\
\hline 215318_at & 27.675882 & CG012 & 116829 & Hypothetical gene CG012 \\
\hline 215917_at & 27.72305 & SNPH & 9751 & Syntaphilin \\
\hline \multirow[t]{5}{*}{ 216039_at } & 28.136038 & LOC100132832 & 100132832 & Postmeiotic segregation increased 2-like 5-like \\
\hline & & LOC441259 & 392713 & PMS2 postmeiotic segregation increased 2 (S. cerevisiae)-like \\
\hline & & LOC730324 & 441259 & Similar to postmeiotic segregation increased 2-like 2 \\
\hline & & PMS2 & 5379 & PMS2 postmeiotic segregation increased 2 (S. cerevisiae) \\
\hline & & PMS2L1 & 5380 & Postmeiotic segregation increased 2-like 1 pseudogene \\
\hline
\end{tabular}


Table I. Continued.

\begin{tabular}{|c|c|c|c|c|}
\hline Probe set ID & Fold change & Gene symbol & Entrez gene & Gene title \\
\hline & & PMS2L12 & 5383 & Postmeiotic segregation increased 2-like 12 pseudogene \\
\hline & & PMS2L2 & 5387 & Postmeiotic segregation increased 2-like 2 pseudogene \\
\hline & & PMS2L3 & 5395 & Postmeiotic segregation increased 2-like 3 \\
\hline & & PMS2L5 & 730324 & Postmeiotic segregation increased 2-like 5 \\
\hline 216614_at & 38.53543 & & & \\
\hline 217546_at & 59.020702 & MT1M & 4499 & Metallothionein $1 \mathrm{M}$ \\
\hline 217664_at & 26.640495 & & & \\
\hline \multirow[t]{2}{*}{ 218720_x_at } & 44.51107 & LOC652900 & 26470 & $\begin{array}{l}\text { Similar to seizure related } 6 \text { homolog (mouse)-like } 2 \\
\text { isoform } 1\end{array}$ \\
\hline & & SEZ6L2 & 652900 & Seizure related 6 homolog (mouse)-like 2 \\
\hline 219396_s_at & 41.20278 & NEIL1 & 79661 & Nei endonuclease VIII-like 1 (E. coli) \\
\hline 219563_at & 28.401752 & $\mathrm{C} 14$ or $\mathrm{f} 139$ & 79686 & Chromosome 14 open reading frame 139 \\
\hline 219727_at & 29.042574 & DUOX2 & 50506 & Dual oxidase 2 \\
\hline 219728_at & 23.185019 & MYOT & 9499 & Myotilin \\
\hline 219768_at & 90.29275 & VTCN1 & 79679 & V-set domain containing $\mathrm{T}$ cell activation inhibitor 1 \\
\hline 219947_at & 43.223457 & CLEC4A & 50856 & C-type lectin domain family 4 , member A \\
\hline 219949_at & 53.802288 & LRRC2 & 79442 & Leucine-rich repeat containing 2 \\
\hline 220086_at & 24.022339 & IKZF5 & 64376 & IKAROS family zinc finger 5 (Pegasus) \\
\hline 220160_s_at & 20.245548 & KPTN & 11133 & Kaptin (actin binding protein) \\
\hline 221195_at & 25.616514 & RNFT1 & 51136 & Ring finger protein, transmembrane 1 \\
\hline 222106_at & 26.959286 & PRND & 23627 & Prion protein 2 (dublet) \\
\hline 222565_s_at & 60.40278 & PRKD3 & 23683 & Protein kinase D3 \\
\hline 222668_at & 114.43788 & KCTD15 & 79047 & Potassium channel tetramerisation domain containing 15 \\
\hline 222720_x_at & 23.61017 & $\mathrm{C} 1$ or $\mathrm{f} 27$ & 54953 & Chromosome 1 open reading frame 27 \\
\hline 223235_s_at & 76.54248 & SMOC2 & 64094 & SPARC related modular calcium binding 2 \\
\hline 223599_at & 21.029135 & TRIM6 & 117854 & Tripartite motif-containing 6 \\
\hline 223985_at & 27.464828 & FSD1L & 83856 & Fibronectin type III and SPRY domain containing 1-like \\
\hline 223999_at & 26.675077 & PPIL2 & 23759 & Peptidylprolyl isomerase (cyclophilin)-like 2 \\
\hline 224098_at & 32.526546 & & & \\
\hline 224348_s_at & 34.73594 & & & \\
\hline 224533_s_at & 77.99536 & C6 or f142 & 90523 & Chromosome 6 open reading frame 142 \\
\hline 225627_s_at & 23.322224 & CACHD1 & 57685 & Cache domain containing 1 \\
\hline 225728_at & 26.672054 & SORBS2 & 8470 & Sorbin and SH3 domain containing 2 \\
\hline 226591_at & 26.339521 & SNRPN & 6638 & Small nuclear ribonucleoprotein polypeptide $\mathrm{N}$ \\
\hline 227491_at & 20.302715 & ELOVL6 & 79071 & $\begin{array}{l}\text { ELOVL family member 6, elongation of long chain fatty } \\
\text { acids (FEN1/Elo2, SUR4/Elo3-like, yeast) }\end{array}$ \\
\hline 227870_at & 48.255272 & IGDCC4 & 57722 & Immunoglobulin superfamily, DCC subclass, member 4 \\
\hline 227925_at & 65.23458 & FLJ39051 & 399972 & Hypothetical gene supported by AK096370 \\
\hline 227955_s_at & 29.176859 & EFNA5 & 1946 & Ephrin-A5 \\
\hline 228007_at & 20.671658 & C6 or f204 & 387119 & Chromosome 6 open reading frame 204 \\
\hline 228072_at & 35.00761 & SYT12 & 91683 & Synaptotagmin XII \\
\hline 229228_at & 178.89961 & CREB5 & 9586 & cAMP responsive element binding protein 5 \\
\hline 229450_at & 20.979269 & IFIT3 & 3437 & $\begin{array}{l}\text { Interferon-induced protein with tetratricopeptide } \\
\text { repeats } 3\end{array}$ \\
\hline 229478_x_at & 48.66807 & BIVM & 54841 & Basic, immunoglobulin-like variable motif containing \\
\hline 229518_at & 24.889814 & FAM46B & 115572 & Family with sequence similarity 46, member B \\
\hline 229579_s_at & 42.72015 & DISP2 & 85455 & Dispatched homolog 2 (Drosophila) \\
\hline 229725_at & 30.584335 & ACSL6 & 23305 & Acyl-CoA synthetase long-chain family member 6 \\
\hline 230245_s_at & 25.038433 & LOC283663 & 283663 & Hypothetical LOC283663 \\
\hline \multirow[t]{2}{*}{ 230487_at } & 66.86591 & C6 or f99 & 100130967 & Chromosome 6 open reading frame 99 \\
\hline & & LOC100130967 & 389440 & Similar to hCG2044932 \\
\hline 230615_at & 46.127205 & DUOXA2 & 405753 & Dual oxidase maturation factor 2 \\
\hline 230660_at & 37.561954 & SERTAD4 & 56256 & SERTA domain containing 4 \\
\hline 230781_at & 42.71768 & & & \\
\hline 231102_at & 32.751915 & CROT & 54677 & Carnitine O-octanoyltransferase \\
\hline 231203_at & 35.097168 & LOC100129633 & 100129633 & Similar to hCG1651427 \\
\hline 231336_at & 55.55161 & CPNE4 & 131034 & Copine IV \\
\hline 231598_x_at & 111.41086 & & & \\
\hline 231899_at & 30.031355 & $\mathrm{ZC} 3 \mathrm{H} 12 \mathrm{C}$ & 85463 & Zinc finger CCCH-type containing $12 \mathrm{C}$ \\
\hline 232068_s_at & 20.96932 & TLR4 & 7099 & Toll-like receptor 4 \\
\hline 232387_at & 23.740568 & AP1GBP1 & 11276 & AP1 $\gamma$ subunit binding protein 1 \\
\hline 232585_at & 24.154518 & LOC100128729 & 100128729 & Similar to TLK2 protein \\
\hline
\end{tabular}


Table I. Continued

\begin{tabular}{|c|c|c|c|c|}
\hline Probe set ID & Fold change & Gene symbol & Entrez gene & Gene title \\
\hline & & LOC100134651 & 100134651 & Similar to TLK2 protein \\
\hline & & LOC731082 & 11011 & $\begin{array}{l}\text { Similar to serine/threonine-protein kinase tousled-like } 2 \\
\text { (tousled-like kinase 2) }\end{array}$ \\
\hline & & TLK2 & 731082 & Tousled-like kinase 2 \\
\hline 233149_at & 20.532196 & & & \\
\hline 233372_at & 48.244698 & & & \\
\hline 233884_at & 21.642752 & HIVEP3 & 59269 & $\begin{array}{l}\text { Human immunodeficiency virus type I enhancer } \\
\text { binding protein } 3\end{array}$ \\
\hline 233902_at & 35.82076 & GUCA1C & 9626 & Guanylate cyclase activator $1 \mathrm{C}$ \\
\hline 233984_at & 22.362148 & & & \\
\hline 234700_s_at & 146.27199 & RNASE7 & 84659 & Ribonuclease, RNase A family, 7 \\
\hline 234980_at & 55.37136 & TMEM56 & 148534 & Transmembrane protein 56 \\
\hline 235795_at & 27.020695 & PAX6 & 5080 & Paired box 6 \\
\hline 236302_at & 21.677221 & PPM1E & 22843 & Protein phosphatase 1E (PP2C domain containing) \\
\hline 236365_at & 31.352936 & & & \\
\hline 236522_at & 25.091562 & & & \\
\hline 236542_at & 42.33878 & & & \\
\hline 236740_at & 25.037415 & & & \\
\hline 236801_at & 50.310337 & & & \\
\hline 237250_at & 49.111843 & & & \\
\hline 237406_at & 28.270689 & NEK6 & 10783 & NIMA (never in mitosis gene a)-related kinase 6 \\
\hline 237950_s_at & 49.878513 & & & \\
\hline 237974_at & 36.01243 & ABHD12B & 145447 & Abhydrolase domain containing $12 \mathrm{~B}$ \\
\hline 237996_at & 54.733566 & & & \\
\hline 238088_at & 20.880829 & LOC100129194 & 100129194 & Hypothetical protein LOC100129194 \\
\hline 238363_at & 29.599056 & & & \\
\hline 238520_at & 45.876377 & TRERF1 & 55809 & Transcriptional regulating factor 1 \\
\hline 238611_at & 22.46198 & & & \\
\hline 239381_at & 61.001457 & KLK7 & 5650 & Kallikrein-related peptidase 7 \\
\hline 240214_at & 27.089334 & & & \\
\hline 241044_x_at & 37.12408 & & & \\
\hline 241579_at & 40.58749 & & & \\
\hline 241814_at & 28.057734 & & & \\
\hline 241842_x_at & 27.38526 & $\mathrm{C} 19$ or $\mathrm{f} 45$ & 374877 & Chromosome 19 open reading frame 45 \\
\hline 242003_at & 35.487854 & ERICH1 & 157697 & Glutamate-rich 1 \\
\hline 242138_at & 27.004044 & DLX1 & 1745 & Distal-less homeobox 1 \\
\hline 242249_at & 21.104935 & & & \\
\hline 242316_at & 25.703829 & & & \\
\hline 242509_at & 26.935835 & & & \\
\hline 242769_at & 26.859947 & & & \\
\hline 242791_at & 26.823769 & FBXO3 & 26273 & F-box protein 3 \\
\hline 242893_at & 28.790308 & & & \\
\hline 243753_at & 20.372232 & $\mathrm{C} 1$ or $\mathrm{f} 173$ & 127254 & Chromosome 1 open reading frame 173 \\
\hline 244016_at & 41.27944 & & & \\
\hline 244639_at & 20.593792 & & & \\
\hline 244785_at & 25.557535 & & & \\
\hline 1552480_s_at & 27.07572 & PTPRC & 5788 & Protein tyrosine phosphatase, receptor type, C \\
\hline 1552580_at & 30.773138 & TRIML2 & 205860 & Tripartite motif family-like 2 \\
\hline 1552904_at & 26.276854 & NETO1 & 81832 & Neuropilin (NRP) and tolloid (TLL)-like 1 \\
\hline 1552987_a_at & 41.45497 & MGC42157 & 439933 & Hypothetical locus MGC42157 \\
\hline 1553037_a_at & 22.476143 & SYN2 & 6854 & Synapsin II \\
\hline 1553315_at & 22.418777 & SLFNL1 & 200172 & Schlafen-like 1 \\
\hline 1553635_s_at & 22.641033 & TCTEX1D1 & 200132 & Tctex 1 domain containing 1 \\
\hline 1553789_a_at & 27.130203 & $\mathrm{C} 21$ or $\mathrm{f} 58$ & 54058 & Chromosome 21 open reading frame 58 \\
\hline 1554001_at & 49.381943 & TRIM37 & 4591 & Tripartite motif-containing 37 \\
\hline 1554398_at & 27.990637 & LYG2 & 254773 & Lysozyme G-like 2 \\
\hline 1554512_a_at & 40.911217 & $\mathrm{CCDC} 123$ & 84902 & Coiled-coil domain containing 123 \\
\hline 1555300_a_at & 25.510637 & MED12L & 116931 & Mediator complex subunit 12-like \\
\hline 1555717_at & 38.556057 & & & \\
\hline 1555912_at & 33.604454 & ST7OT1 & 93653 & ST7 overlapping transcript 1 (non-protein coding) \\
\hline 1556008_a_at & 36.32589 & & & \\
\hline 1556202_at & 39.568478 & SRGAP2 & 23380 & SLIT-ROBO Rho GTPase activating protein 2 \\
\hline 1556541_s_at & 29.452276 & & & \\
\hline
\end{tabular}


Table I. Continued.

\begin{tabular}{|c|c|c|c|c|}
\hline Probe set ID & Fold change & Gene symbol & Entrez gene & Gene title \\
\hline 1558552_s_at & 20.966536 & $\mathrm{C} 3$ or $\mathrm{f} 55$ & 152078 & Chromosome 3 open reading frame 55 \\
\hline 1558234_at & 31.376406 & FLJ36644 & 400617 & Hypothetical protein LOC400617 \\
\hline 1558791_at & 43.679718 & LOC286467 & 286467 & Hypothetical LOC286467 \\
\hline 1561114_a_at & 22.05421 & DEPDC4 & 120863 & DEP domain containing 4 \\
\hline 1560048 at & 27.137468 & & & \\
\hline 1561101_at & 35.96605 & LOC153469 & 153469 & $\begin{array}{l}\text { Hypothetical protein LOC153469 1561260_at } \\
23.416117\end{array}$ \\
\hline 1561573_at & 23.90905 & & & \\
\hline 1561714_a_at & 41.711376 & B3GALNT2 & 148789 & $\beta$-1,3-N-acetylgalactosaminyltransferase 2 \\
\hline 1561731_at & 49.069042 & & & \\
\hline 1561918_at & 21.02261 & & & \\
\hline 1562860_at & 40.932205 & & & \\
\hline 1562990_at & 26.946367 & & & \\
\hline 1563392_at & 23.033009 & & & \\
\hline \multirow[t]{2}{*}{ 1563456_at } & 25.698204 & CCDC141 & 285025 & Coiled-coil domain containing 141 \\
\hline & & LOC 285026 & 285026 & Hypothetical protein LOC285026 \\
\hline 1564653_s_at & 35.795006 & LEKR1 & 389170 & Leucine, glutamate and lysine rich 1 \\
\hline 1564932_at & 21.246243 & & & \\
\hline 1565337_at & 22.219374 & DNAH6 & 1768 & Dynein, axonemal, heavy chain 6 \\
\hline 1568941_a_at & 24.125954 & & & \\
\hline 1569408 at & 27.472164 & EIF2C4 & 192670 & Eukaryotic translation initiation factor $2 \mathrm{C}, 4$ \\
\hline 1569599_at & 47.001877 & SAMSN1 & 64092 & $\begin{array}{l}\text { SAM domain, SH3 domain and nuclear localization } \\
\text { signals } 1\end{array}$ \\
\hline 1569772_x_at & 23.892517 & & & \\
\hline 1569832_at & 34.25927 & hCG_2045206 & 100131655 & Hypothetical LOC100131655 \\
\hline 1569948_at & 21.763708 & & & \\
\hline 1570076_at & 30.77381 & & & \\
\hline
\end{tabular}

patient with lymphatic and hepatic metastases. Samples were snap-frozen and stored in liquid nitrogen until use. Prior written informed consent was obtained from the patient and the study received ethics board approval at the First Affiliated Hospital, College of Medicine, Zhejiang University, Hangzhou, China. The patient had not received chemotherapy or radiation therapy prior to surgery. The histological type was determined according to the WHO criteria. The tumor was diagnosed as mucinous adenocarcinoma (T3N2M1).

Genome-wide gene expression analysis. RNA was extracted from tumor specimens. Total RNA of homogenized tumor samples was prepared with TRIzol RNA extraction reagent (Invitrogen) followed by purification using the RNeasy Mini kit (Qiagen) according to the manufacturer's instructions. A DNase I (Qiagen) digestion step was included to eliminate genomic DNA. The quality of the total RNA was examined for integrity using RNA LabChips and the Agilent Bioanalyzer 2100 (Agilent Technologies), and the concentration was measured using the Peqlab NanoDrop. Only RNA with an RNA integrity number $>6.5$ was used for cDNA synthesis. The one-cycle eukaryotic target labeling assay from Affymetrix was used according to manufacturer's instructions as previously described $(16,17)$. Data analysis was also performed as previously described $(16,17)$.

Statistical analysis. Hierarchical clustering of all microarray experiments was performed based on all 54,675 probe sets represented on the HGU133Plus2.0 array (Affymetrix)
$(\mathrm{P}<0.04)$ using positive correlation and complete linkage. Gene expression of the primary tumor was compared with the median arrays of the paired lymphatic metastatic and hepatic metastatic tumors from each xenograft model in a paired t-test.

\section{Results}

Heterogeneity in primary colon carcinoma and its paired lymphatic and hepatic metastases. Based on the results of the genome-wide gene expression analysis, a paired t-test between the primary colon carcinoma and its paired lymphatic metastasis revealed 190 differentially expressed probe sets with a fold change $\geq 20$. Clustering based on these 190 probe sets demonstrated a clear distinction between primary colon carcinoma and its paired lymphatic metastasis, with the majority of the probe sets (115 of 190) being downregulated in the lymphatic metastasis and only a small number being upregulated (75 of 190) (Table I). A paired t-test between the primary colon carcinoma and its paired hepatic metastasis revealed 150 differentially expressed probe sets with a fold change $\geq 20$. Clustering based on these 150 probe sets demonstrated a clear distinction between primary colon carcinoma and its paired hepatic metastasis, with the majority of probe sets (94 of 150) being downregulated in the hepatic metastasis and only a very small number being upregulated (56 of 150) (Table II). A paired t-test between colon carcinoma lymphatic metastasis and hepatic metastasis revealed 176 differentially expressed probe sets with a fold change of only $\geq 10$. Clustering based on these 
Table II.Genes differentially expressed in the primary colon carcinoma specimen and its paired hepatic metastasis.

\begin{tabular}{|c|c|c|c|c|}
\hline Probe set ID & Fold change & Gene symbol & Entrez gene & Gene title \\
\hline 201744_s_at & 109.43063 & LUM & 4060 & Lumican \\
\hline 203639_s_at & 39.816193 & FGFR2 & 2263 & Fibroblast growth factor receptor 2 \\
\hline 203687_at & 23.863962 & CX3CL1 & 6376 & Chemokine (C-X3-C motif) ligand 1 \\
\hline 203697_at & 55.182484 & FRZB & 2487 & Frizzled-related protein \\
\hline 203817_at & 36.81657 & GUCY1B3 & 2983 & Guanylate cyclase 1 , soluble, $\beta 3$ \\
\hline 204010_s_at & 40.820312 & KRAS & 3845 & v-Ki-ras2 Kirsten rat sarcoma viral oncogene homolog \\
\hline 204439_at & 50.40096 & IFI44L & 10964 & Interferon-induced protein 44-like \\
\hline 204646_at & 73.7989 & DPYD & 1806 & Dihydropyrimidine dehydrogenase \\
\hline 204704_s_at & 30.57162 & ALDOB & 229 & Aldolase B, fructose-bisphosphate \\
\hline 205830_at & 40.507374 & CLGN & 1047 & Calmegin \\
\hline 205893_at & 33.37443 & NLGN1 & 22871 & Neuroligin 1 \\
\hline 206091_at & 43.29831 & MATN3 & 4148 & Matrilin 3 \\
\hline 206218_at & 29.100803 & MAGEB2 & 4113 & Melanoma antigen family B, 2 \\
\hline 207349_s_at & 24.040384 & UCP3 & 7352 & Uncoupling protein 3 (mitochondrial, proton carrier) \\
\hline 207583_at & 27.094574 & $\mathrm{ABCD} 2$ & 225 & ATP-binding cassette, sub-family D (ALD), member 2 \\
\hline 207750_at & 29.539001 & EPS15L2 & 55380 & Epidermal growth factor receptor pathway substrate 15 -like 2 \\
\hline 208250_s_at & 34.10199 & DMBT1 & 1755 & Deleted in malignant brain tumors 1 \\
\hline 210077_s_at & 23.053602 & SFRS5 & 6430 & Splicing factor, arginine/serine-rich 5 \\
\hline 210143_at & 36.192024 & ANXA10 & 11199 & Annexin A10 \\
\hline 210510_s_at & 52.630417 & NRP1 & 8829 & Neuropilin 1 \\
\hline 210571_s_at & 69.8555 & CMAH & 8418 & $\begin{array}{l}\text { Cytidine monophosphate-N-acetylneuraminic acid hydroxylase } \\
\text { (CMP-N-acetylneuraminate monooxygenase) pseudogene }\end{array}$ \\
\hline 211204_at & 21.602095 & ME1 & 4199 & Malic enzyme $1, \mathrm{NADP}(+)$-dependent, cytosolic \\
\hline 211842_s_at & 20.652378 & SLC24A1 & 9187 & $\begin{array}{l}\text { Solute carrier family } 24 \text { (sodium/potassium/calcium exchanger), } \\
\text { member } 1\end{array}$ \\
\hline 212768_s_at & 68.42675 & OLFM4 & 10562 & Olfactomedin 4 \\
\hline 213496_at & 31.919434 & LPPR4 & 9890 & Plasticity related gene 1 \\
\hline 213519_s_at & 26.87308 & LAMA2 & 3908 & Laminin, $\alpha 2$ \\
\hline 214044_at & 35.559616 & RYR2 & 6262 & Ryanodine receptor 2 (cardiac) \\
\hline 214121_x_at & 29.512691 & PDLIM7 & 9260 & PDZ and LIM domain 7 (enigma) \\
\hline 214146_s_at & 50.72601 & PPBP & 5473 & Pro-platelet basic protein [chemokine (C-X-C motif) ligand 7] \\
\hline \multirow[t]{2}{*}{$214567 \_s \_a t$} & 24.816338 & XCL1 & 6375 & Chemokine (C motif) ligand 1 \\
\hline & & XCL2 & 6846 & Chemokine (C motif) ligand 2 \\
\hline 214823_at & 45.280537 & ZNF204 & 7754 & Zinc finger protein 204 pseudogene \\
\hline \multirow[t]{2}{*}{ 215172_at } & 35.013386 & PTPN20A & 26095 & Protein tyrosine phosphatase, non-receptor type $20 \mathrm{~A}$ \\
\hline & & PTPN20B & 653129 & Protein tyrosine phosphatase, non-receptor type $20 \mathrm{~B}$ \\
\hline \multirow[t]{2}{*}{ 216236_s_at } & 22.056973 & SLC2A14 & 144195 & Solute carrier family 2 (facilitated glucose transporter) \\
\hline & & SLC2A3 & 6515 & $\begin{array}{l}\text { Member } 14 / \text { solute carrier family } 2 \text { (facilitated glucose transporter) } \\
\text { member } 3\end{array}$ \\
\hline 216918_s_at & 22.751093 & DST & 667 & Dystonin \\
\hline 217549_at & 33.947136 & & & \\
\hline 217584_at & 28.199327 & & & \\
\hline 219396_s_at & 51.44716 & NEIL1 & 79661 & Nei endonuclease VIII-like 1 (E. coli) \\
\hline 219938_s_at & 38.966156 & PSTPIP2 & 9050 & Proline-serine-threonine phosphatase interacting protein 2 \\
\hline 219947_at & 42.138695 & CLEC4A & 50856 & C-type lectin domain family 4 , member A \\
\hline 219949_at & 40.146168 & LRRC2 & 79442 & Leucine-rich repeat containing 2 \\
\hline 220253_s_at & 49.8748 & LRP12 & 29967 & Low density lipoprotein-related protein 12 \\
\hline 220333_at & 24.75799 & PAQR5 & 54852 & Progestin and adipoQ receptor family member $\mathrm{V}$ \\
\hline 220858_at & 40.026302 & SORBS2 & 8470 & Sorbin and SH3 domain containing 2 \\
\hline 222249_at & 32.831154 & & & \\
\hline 222664_at & 57.42625 & KCTD15 & 79047 & Potassium channel tetramerisation domain containing 15 \\
\hline 222720_x_at & 37.01965 & $\mathrm{C} 1$ or $\mathrm{f} 27$ & 54953 & Chromosome 1 open reading frame 27 \\
\hline 222738_at & 49.0615 & WWC2 & 80014 & WW and $\mathrm{C} 2$ domain containing 2 \\
\hline 223235_s_at & 83.11333 & SMOC2 & 64094 & SPARC related modular calcium binding 2 \\
\hline 223553_s_at & 60.20032 & DOK3 & 79930 & Docking protein 3 \\
\hline 223645_s_at & 23.843172 & CYorf15B & 84663 & Chromosome $\mathrm{Y}$ open reading frame $15 \mathrm{~B}$ \\
\hline 223985_at & 25.144203 & FSD1L & 83856 & Fibronectin type III and SPRY domain containing 1-like \\
\hline 223999_at & 43.358845 & PPIL2 & 23759 & Peptidylprolyl isomerase (cyclophilin)-like 2 \\
\hline 224029_x_at & 53.921314 & SCN11A & 11280 & Sodium channel, voltage-gated, type XI, $\alpha$ subunit \\
\hline 224135_at & 28.728426 & WDR87 & 83889 & WD repeat domain 87 \\
\hline 224370_s_at & 51.475292 & CAPS2 & 84698 & Calcyphosine 2 \\
\hline 224533_s_at & 75.15053 & C6 or $\mathrm{f} 142$ & 90523 & Chromosome 6 open reading frame 142 \\
\hline 225627_s_at & 20.237694 & CACHD1 & 57685 & Cache domain containing 1 \\
\hline
\end{tabular}


Table II. Continued.

\begin{tabular}{|c|c|c|c|c|}
\hline Probe set ID & Fold change & Gene symbol & Entrez gene & Gene title \\
\hline 225728_at & 45.161 & SORBS2 & 8470 & Sorbin and SH3 domain containing 2 \\
\hline 226147_s_at & 64.73383 & PIGR & 5284 & Polymeric immunoglobulin receptor \\
\hline 226425_at & 21.041115 & CLIP4 & 79745 & CAP-GLY domain containing linker protein family, member 4 \\
\hline 226435_at & 23.594818 & PAPLN & 89932 & Papilin, proteoglycan-like sulfated glycoprotein \\
\hline 226591_at & 29.041143 & SNRPN & 6638 & Small nuclear ribonucleoprotein polypeptide $\mathrm{N}$ \\
\hline 227826_s_at & 47.852997 & & & \\
\hline 227925_at & 29.181805 & FLJ39051 & 399972 & Hypothetical gene supported by AK096370 \\
\hline 228007_at & 23.74942 & $\mathrm{C} 6$ or $\mathrm{f} 204$ & 387119 & Chromosome 6 open reading frame 204 \\
\hline 228255_at & 23.693722 & ALS2CR4 & 65062 & $\begin{array}{l}\text { Amyotrophic lateral sclerosis } 2 \text { (juvenile) chromosome region, } \\
\text { candidate } 4\end{array}$ \\
\hline 228766_at & 35.04908 & CD36 & 948 & CD36 molecule (thrombospondin receptor) \\
\hline 229495_at & 24.522652 & PM20D2 & 135293 & Peptidase M20 domain containing 2 \\
\hline 230245_s_at & 35.8261 & LOC283663 & 283663 & Hypothetical LOC283663 \\
\hline 230720_at & 20.412619 & RNF182 & 221687 & Ring finger protein 182 \\
\hline 231102_at & 126.71907 & CROT & 54677 & Carnitine O-octanoyltransferase \\
\hline 231114_at & 49.288345 & SPATA22 & 84690 & Spermatogenesis associated 22 \\
\hline 231385_at & 37.57739 & DPPA3 & 359787 & Developmental pluripotency associated 3 \\
\hline 231560_at & 24.556393 & & & \\
\hline 231969_at & 32.270084 & STOX2 & 56977 & Storkhead box 2 \\
\hline 232049_at & 20.035376 & & & \\
\hline 232090_at & 21.041267 & LOC100128178 & 100128178 & Similar to hCG2041313 \\
\hline 232397_at & 116.284195 & & & \\
\hline 232481_s_at & 28.357405 & SLITRK6 & 84189 & SLIT and NTRK-like family, member 6 \\
\hline 232641_at & 30.381804 & ZNF596 & 169270 & Zinc finger protein 596 \\
\hline 233721_x_at & 38.845203 & & & \\
\hline 233814_at & 50.578884 & EFNA5 & 1946 & Ephrin-A5 \\
\hline 233871_at & 42.2906 & & & \\
\hline 233934_at & 24.12915 & LOC349160 & 349160 & Hypothetical protein LOC349160 \\
\hline 234030_at & 36.926117 & & & \\
\hline 234980_at & 21.773212 & TMEM56 & 148534 & Transmembrane protein 56 \\
\hline 235306_at & 22.31179 & GIMAP8 & 155038 & GTPase, IMAP family member 8 \\
\hline 236220_at & 39.285076 & & & \\
\hline 236365_at & 38.961994 & & & \\
\hline 236698_at & 21.145327 & & & \\
\hline 236740_at & 21.301474 & & & \\
\hline 237150_at & 21.254215 & & & \\
\hline 237427_at & 20.181032 & & & \\
\hline 237905_at & 23.934755 & KRT25 & 147183 & Keratin 25 \\
\hline 238009_at & 20.990005 & & & \\
\hline 238751_at & 38.94119 & & & \\
\hline 239443_at & 26.224146 & PCDHB6 & 56130 & Protocadherin $\beta 6$ \\
\hline 239447_at & 44.547733 & TRA2B & 6434 & Transformer $2 \beta$ homolog (Drosophila) \\
\hline 239477_at & 24.663624 & EFHB & 151651 & EF-hand domain family, member B \\
\hline 239698_at & 24.539743 & & & \\
\hline 240120_at & 20.922508 & & & \\
\hline 240214_at & 31.374973 & & & \\
\hline 240466_at & 24.025442 & & & \\
\hline 240964_at & 21.602964 & & & \\
\hline 241829_at & 27.049297 & FAM124A & 220108 & Family with sequence similarity $124 \mathrm{~A}$ \\
\hline 241926_s_at & 51.05444 & ERG & 2078 & v-ets erythroblastosis virus E26 oncogene homolog (avian) \\
\hline 242003_at & 20.770477 & ERICH1 & 157697 & Glutamate-rich 1 \\
\hline 242249_at & 41.35373 & & & \\
\hline 242340_at & 33.86683 & & & \\
\hline 242721_at & 46.80031 & & & \\
\hline 242893_at & 42.70509 & & & \\
\hline 243500_at & 30.1103 & CASD1 & 64921 & CAS 1 domain containing 1 \\
\hline 243746_at & 45.71318 & IGHMBP2 & 3508 & Immunoglobulin mu binding protein 2 \\
\hline 243901_at & 28.134262 & ALDOB & 229 & Aldolase B, fructose-bisphosphate \\
\hline 244531_at & 61.498493 & NNT & 23530 & Nicotinamide nucleotide transhydrogenase \\
\hline 1552835_at & 21.108583 & DENND1B & 163486 & DENN/MADD domain containing 1B \\
\hline 1552927_at & 22.232943 & MAP3K7IP3 & 257397 & $\begin{array}{l}\text { Mitogen-activated protein kinase kinase kinase } 7 \text { interacting } \\
\text { protein } 3\end{array}$ \\
\hline 1552999_a_at & 27.387323 & WFDC10B & 280664 & WAP four-disulfide core domain $10 \mathrm{~B}$ \\
\hline
\end{tabular}


Table II. Continued.

\begin{tabular}{|c|c|c|c|c|}
\hline Probe set ID & Fold change & Gene symbol & Entrez gene & Gene title \\
\hline 1554176_a_at & 23.11273 & $\mathrm{C} 3$ or $\mathrm{f} 33$ & 285315 & Chromosome 3 open reading frame 33 \\
\hline 1554001_at & 41.838184 & TRIM37 & 4591 & Tripartite motif-containing 37 \\
\hline 1554398_at & 24.453304 & LYG2 & 254773 & Lysozyme G-like 2 \\
\hline 1555912_at & 44.85555 & ST7OT1 & 93653 & ST7 overlapping transcript 1 (non-protein coding) \\
\hline 1556008_a_at & 47.270073 & & & \\
\hline 1556203_a_at & $30 . X 93089$ & SRGAP2 & 23380 & SLIT-ROBO Rho GTPase activating protein 2 \\
\hline 1556289_at & 20.807093 & & & \\
\hline 1556357_s_at & 26.665522 & ERICH1 & 157697 & Glutamate-rich 1 \\
\hline 1556392_a_at & 22.948168 & & & \\
\hline 1556393 at & 36.13576 & & & \\
\hline 1557520_a_at & 24.872932 & & & \\
\hline 1558135_at & 25.871822 & TAF11 & 6882 & $\begin{array}{l}\text { TAF11 RNA polymerase II, TATA box binding protein } \\
\text { (TBP)-associated factor, } 28 \mathrm{kDa}\end{array}$ \\
\hline 1558234_at & 55.475685 & FLJ36644 & 400617 & Hypothetical protein LOC400617 \\
\hline 1558574_at & 26.385082 & & & \\
\hline 1558606_s_at & 20.5551 & & & \\
\hline 1558693_s_at & 29.909445 & $\mathrm{C} 1$ or $\mathrm{f} 85$ & 112770 & Chromosome 1 open reading frame 85 \\
\hline 1558815_at & 49.138927 & SORBS2 & 8470 & Sorbin and SH3 domain containing 2 \\
\hline 1558934_a_at & 37.3662 & GTF2H5 & 404672 & General transcription factor IIH, polypeptide 5 \\
\hline 1560048_at & 32.75761 & & & \\
\hline 1560422_at & 45.097427 & & & \\
\hline 1561432_at & 23.945683 & & & \\
\hline 1561882_at & 42.562286 & SYTL3 & 94120 & Synaptotagmin-like 3 \\
\hline 1562094_at & 28.180384 & & & \\
\hline 1562294_x_at & 22.353355 & ANKRD30B & 374860 & Ankyrin repeat domain $30 \mathrm{~B}$ \\
\hline 1562310_at & 22.610895 & & & \\
\hline 1562472_at & 26.148716 & & & \\
\hline 1562742_at & 59.214592 & & & \\
\hline 1562860_at & 21.426584 & & & \\
\hline 1563112_at & 76.9244 & FAM120AOS & 158293 & Family with sequence similarity $120 \mathrm{~A}$ opposite strand \\
\hline 1564039_at & 24.121265 & ZSCAN23 & 222696 & Zinc finger and SCAN domain containing 23 \\
\hline 1568012_at & 31.55809 & CLIP1 & 6249 & CAP-GLY domain containing linker protein 1 \\
\hline 1570600_at & 22.040146 & & & \\
\hline
\end{tabular}

176 probe sets demonstrated a clear distinction between colon carcinoma lymphatic metastasis and hepatic metastasis, with the majority of probe sets (114 of 176) being downregulated in the hepatic metastasis and only a very small number being upregulated (62 of 176) (Table III).

\section{Discussion}

The purpose of this study was to investigate the heterogeneity in primary colon carcinoma and its corresponding lymphatic and hepatic metastases using GeneChip HGU133Plus2.0 expression arrays (Affymetrix). Our results demonstrate that the genome-wide gene expression varies between primary colon carcinoma and its paired lymphatic and hepatic metastases. In our study, we firstly demonstrate that a high degree of heterogeneity in gene expression exists in primary colon carcinoma and corresponding lymphatic and hepatic metastases.

The main purpose of investigating the heterogeneity found in primary tumors and corresponding metastases is to evaluate the effect of such heterogeneity on the efficacy of anticancer therapy and cancer patient prognosis. As previously discussed $(1,16,18)$, the patient-derived tumor tissue (PDTT) xenograft model, which has a sound establishing method and a retained similarity to the corresponding original donor tumors in histological presentation and biological behavior, including protein expression, tumor biomarker status and genomic and genetic status, has the potential to be an ideal strategy to achieve our purpose. In our previous study, we successfully established PDTT xenograft models of colon carcinoma with lymphatic and hepatic metastases using the same tissue samples from three above-mentioned tumor sites (16). The ideal biological characteristics of such PDTT xenograft models, as previously described (16), led to the consideration that such PDTT models may aid in the investigation of the underlying mechanism of heterogeneity-related anticancer therapy response differences in primary colon carcinoma and corresponding lymphatic and hepatic metastases.

In conclusion, in this study, we investigated the heterogeneity in primary colon carcinoma and corresponding lymphatic and hepatic metastases focusing on genome-wide gene expression, and revealed that there were differences in primary colon carcinoma and matched lymphatic and hepatic metastases. In subsequent studies, further investigation is required to ascertain the heterogeneity of functional genes found in primary colon carcinoma and paired lymphatic and hepatic metastases in a larger group of patients with metastatic 
Table III. Genes differentially expressed in the colon carcinoma lymphatic metastasis specimen and the hepatic metastasis specimen.

\begin{tabular}{|c|c|c|c|c|}
\hline Probe set ID & Fold change & Gene symbol & Entrez gene & Gene title \\
\hline 203323_at & 17.572113 & CAV2 & 858 & Caveolin 2 \\
\hline 203324_s_at & 12.488382 & CAV2 & 858 & Caveolin 2 \\
\hline 203372_s_at & 38.86711 & SOCS2 & 8835 & Suppressor of cytokine signaling 2 \\
\hline 203543_s_at & 11.408775 & KLF9 & 687 & Kruppel-like factor 9 \\
\hline 203561_at & 28.196625 & FCGR2A & 2212 & Fc fragment of IgG, low affinity IIa, receptor (CD32) \\
\hline 203835_at & 11.724807 & LRRC32 & 2615 & Leucine-rich repeat containing 32 \\
\hline 204035_at & 22.833523 & SCG2 & 7857 & Secretogranin II (chromogranin C) \\
\hline 204069_at & 18.195318 & MEIS1 & 4211 & Meis homeobox 1 \\
\hline 204682_at & 12.34678 & LTBP2 & 4053 & Latent transforming growth factor $\beta$ binding protein 2 \\
\hline 204802_at & 34.692444 & RRAD & 6236 & Ras-related associated with diabetes \\
\hline 205610_at & 55.83588 & MYOM1 & 8736 & Myomesin 1, $185 \mathrm{kDa}$ \\
\hline 205692_s_at & 10.167213 & CD38 & 952 & CD38 molecule \\
\hline 205943_at & 17.091461 & TDO2 & 6999 & Tryptophan 2,3-dioxygenase \\
\hline 206022_at & 31.875471 & NDP & 4693 & Norrie disease (pseudoglioma) \\
\hline 206025_s_at & 11.98506 & TNFAIP6 & 7130 & Tumor necrosis factor, $\alpha$-induced protein 6 \\
\hline 206143_at & 17.525316 & SLC26A3 & 1811 & Solute carrier family 26 , member 3 \\
\hline 206488_s_at & 21.102892 & CD36 & 948 & CD36 molecule (thrombospondin receptor) \\
\hline 206654_s_at & 10.075909 & POLR3G & 10622 & Polymerase (RNA) III (DNA directed) polypeptide G (32 kD) \\
\hline 207089_at & 34.273483 & NRAP & 4892 & Nebulin-related anchoring protein \\
\hline 207501_s_at & 19.537613 & FGF12 & 2257 & Fibroblast growth factor 12 \\
\hline 207580_at & 22.841743 & MAGEB4 & 4115 & Melanoma antigen family B, 4 \\
\hline 208086_s_at & 25.490797 & DMD & 1756 & Dystrophin \\
\hline 208259_x_at & 18.155922 & IFNA7 & 3444 & Interferon, $\alpha 7$ \\
\hline 209576_at & 32.88002 & GNAI1 & 2770 & $\begin{array}{l}\text { Guanine nucleotide binding protein (G protein), } \alpha \text { inhibiting activity } \\
\text { polypeptide } 1\end{array}$ \\
\hline 209652_s_at & 12.313976 & PGF & 5228 & Placental growth factor \\
\hline 209700_x_at & 25.640623 & PDE4DIP & 9659 & Phosphodiesterase 4D interacting protein \\
\hline 209776_s_at & 13.3636875 & SLC19A1 & 6573 & Solute carrier family 19 (folate transporter), member 1 \\
\hline 209821_at & 20.940786 & IL33 & 90865 & Interleukin 33 \\
\hline 210077_s_at & 24.33262 & SFRS5 & 6430 & Splicing factor, arginine/serine-rich 5 \\
\hline 210948_s_at & 21.301382 & LEF1 & 51176 & Lymphoid enhancer-binding factor 1 \\
\hline 211190_x_at & 22.055872 & CD84 & 8832 & CD84 molecule \\
\hline 213423_x_at & 10.081812 & TUSC3 & 7991 & Tumor suppressor candidate 3 \\
\hline 213428_s_at & 13.616949 & COL6A1 & 1291 & Collagen, type VI, $\alpha 1$ \\
\hline 214998_at & 31.85507 & AAK1 & 22848 & AP2 associated kinase 1 \\
\hline 215495_s_at & 14.478629 & SAMD4A & 23034 & Sterile $\alpha$ motif domain containing 4A \\
\hline 215513_at & 17.604755 & HYMAI & 57061 & Hydatidiform mole associated and imprinted (non-protein coding) \\
\hline 215657_at & 12.403633 & SLC26A3 & 1811 & Solute carrier family 26 , member 3 \\
\hline 216466_at & 13.427488 & NAV3 & 89795 & Neuron navigator 3 \\
\hline 216589_at & 10.48587 & LOC390998 & 390998 & Similar to hCG1644589 \\
\hline 216695_s_at & 18.244976 & TNKS & 8658 & Tankyrase, TRF1-interacting ankyrin-related ADP-ribose polymerase \\
\hline 216757_at & 10.563152 & & & \\
\hline \multirow[t]{5}{*}{ 217157_x_at } & 15.075894 & IGK & 3514 & Immunoglobulin $\kappa$ locus \\
\hline & & IGKC & 50802 & Immunoglobulin $\kappa$ constant \\
\hline & & LOC647506 & 647506 & Similar to Ig $\kappa$ chain V-I region HK101 precursor \\
\hline & & LOC650405 & 650405 & Similar to Ig $\kappa$ chain V-I region HK102 precursor \\
\hline & & LOC652493 & 652493 & Similar to Ig $\kappa$ chain V-I region HK 102 precursor \\
\hline 217321_x_at & 28.0056 & ATXN3 & 4287 & Ataxin 3 \\
\hline 217665_at & 12.379506 & & & \\
\hline 218638_s_at & 17.851858 & SPON2 & 10417 & Spondin 2, extracellular matrix protein \\
\hline 220858_at & 19.423872 & SORBS2 & 8470 & Sorbin and SH3 domain containing 2 \\
\hline 220906_at & 20.039635 & & & \\
\hline 221154_at & 38.946198 & TRIM49 & 57093 & Tripartite motif-containing 49 \\
\hline 221541_at & 11.172807 & CRISPLD2 & 83716 & Cysteine-rich secretory protein LCCL domain containing 2 \\
\hline 221558_s_at & 10.16347 & LEF1 & 51176 & Lymphoid enhancer-binding factor 1 \\
\hline 31835_at & 11.370078 & HRG & 3273 & Histidine-rich glycoprotein \\
\hline 223599_at & 12.111252 & TRIM6 & 117854 & Tripartite motif-containing 6 \\
\hline 223812_at & 13.24775 & FAM178B & 51252 & Family with sequence similarity 178 , member B \\
\hline 223876_at & 11.548148 & SPATA16 & 83893 & Spermatogenesis associated 16 \\
\hline 224024_at & 22.182268 & ERGIC1 & 57222 & Endoplasmic reticulum-golgi intermediate compartment (ERGIC) 1 \\
\hline 224056_at & 17.563913 & & & \\
\hline
\end{tabular}


Table III. Continued.

\begin{tabular}{|c|c|c|c|c|}
\hline Probe set ID & Fold change & Gene symbol & Entrez gene & Gene title \\
\hline 225029_at & 15.641858 & LOC550643 & 550643 & Hypothetical LOC550643 \\
\hline 225288_at & 11.822223 & COL27A1 & 85301 & Collagen, type XXVII, $\alpha 1$ \\
\hline 226147_s_at & 150.0156 & PIGR & 5284 & Polymeric immunoglobulin receptor \\
\hline 226828_s_at & 29.641703 & HEYL & 26508 & Hairy/enhancer-of-split related with YRPW motif-like \\
\hline 227692_at & 20.724081 & GNAI1 & 2770 & $\begin{array}{l}\text { Guanine nucleotide binding protein (G protein), } \alpha \text { inhibiting activity } \\
\text { polypeptide } 1\end{array}$ \\
\hline 228167_at & 34.570217 & KLHL6 & 89857 & Kelch-like 6 (Drosophila) \\
\hline 228275_at & 21.79044 & & & \\
\hline 228547_at & 23.981333 & NRXN1 & 9378 & Neurexin 1 \\
\hline 229051_at & 26.098585 & & & \\
\hline 229085_at & 12.635592 & LRRC3B & 116135 & Leucine-rich repeat containing $3 \mathrm{~B}$ \\
\hline 229199_at & 10.150482 & SCN9A & 6335 & Sodium channel, voltage-gated, type IX, $\alpha$ subunit \\
\hline 229228_at & 52.54796 & CREB5 & 9586 & cAMP responsive element binding protein 5 \\
\hline 229381_at & 11.871673 & $\mathrm{C} 1$ or $\mathrm{f} 64$ & 149563 & Chromosome 1 open reading frame 64 \\
\hline 229518_at & 28.762573 & FAM46B & 115572 & Family with sequence similarity 46 , member B \\
\hline 229659_s_at & 20.485302 & & & \\
\hline 229725_at & 29.211315 & ACSL6 & 23305 & Acyl-CoA synthetase long-chain family member 6 \\
\hline 229968_at & 13.312329 & & & \\
\hline 230261_at & 16.136084 & ST8SIA4 & 7903 & ST8 $\alpha$-N-acetyl-neuraminide $\alpha$-2,8-sialyltransferase 4 \\
\hline 230351_at & 10.333499 & LOC283481 & 283481 & Hypothetical protein LOC283481 \\
\hline 230615_at & 19.222267 & DUOXA2 & 405753 & Dual oxidase maturation factor 2 \\
\hline 230781_at & 28.851234 & & & \\
\hline 230943_at & 13.1987 & SOX17 & 64321 & SRY (sex determining region Y)-box 17 \\
\hline 231749_at & 11.926773 & TIMM13 & 26517 & Translocase of inner mitochondrial membrane 13 homolog (yeast) \\
\hline 231964_at & 27.403822 & & & \\
\hline 232113_at & 42.29946 & & & \\
\hline 232378_at & 14.133439 & SLC5A9 & 200010 & Solute carrier family 5 (sodium/glucose cotransporter), member 9 \\
\hline 232408_at & 11.252221 & ZFYVE28 & 57732 & Zinc finger, FYVE domain containing 28 \\
\hline 232673_at & 15.140463 & LRRFIP2 & 9209 & Leucine-rich repeat (in FLII) interacting protein 2 \\
\hline 233586_s_at & 23.207222 & KLK12 & 43849 & Kallikrein-related peptidase 12 \\
\hline 233934_at & 20.81878 & LOC349160 & 349160 & Hypothetical protein LOC 349160 \\
\hline 234233_s_at & 15.17553 & & & \\
\hline 235334_at & 15.941539 & ST6GALNAC3 & 256435 & $\begin{array}{l}\text { ST6 ( } \alpha \text {-N-acetyl-neuraminyl-2,3- } \beta \text {-galactosyl-1,3)-N- } \\
\text { acetylgalactosaminide } \alpha-2,6 \text {-sialyltransferase } 3\end{array}$ \\
\hline 235764_at & 17.735975 & & & \\
\hline 236105_at & 11.190036 & & & \\
\hline 236191_at & 34.859856 & & & \\
\hline 236762_at & 13.769478 & АТР9B & 374868 & ATPase, class II, type 9B \\
\hline 236801_at & 14.398608 & & & \\
\hline 237452_at & 13.013656 & & & \\
\hline 237608_at & 11.315111 & & & \\
\hline 238281_at & 17.088953 & & & \\
\hline 238283_at & 11.590735 & LOC151658 & 151658 & Hypothetical LOC151658 \\
\hline 238625_at & 27.370302 & C1orf168 & 199920 & Chromosome 1 open reading frame 168 \\
\hline 238634_x_at & 12.341533 & & & \\
\hline 238876_at & 14.355665 & & & \\
\hline 239506_s_at & 30.187277 & LOC 151300 & 151300 & Hypothetical LOC151300 \\
\hline 239823_at & 10.645902 & & & \\
\hline 239883_s_at & 47.341763 & ANO4 & 121601 & Anoctamin 4 \\
\hline 239995_at & 20.010996 & & & \\
\hline 240212_at & 19.915565 & & & \\
\hline 240334_at & 11.40588 & & & \\
\hline 240856_at & 22.449312 & & & \\
\hline 241308_at & 10.37427 & LOC729589 & 729589 & Hypothetical LOC729589 \\
\hline 241765_at & 33.79925 & $\mathrm{CPM}$ & 1368 & Carboxypeptidase $\mathrm{M}$ \\
\hline 241841_at & 16.08523 & & & \\
\hline 242006_at & 12.057802 & LCA5 & 167691 & Leber congenital amaurosis 5 \\
\hline 242034_at & 37.776375 & FBXL17 & 64839 & F-box and leucine-rich repeat protein 17 \\
\hline 242193_at & 10.155277 & LOC100130155 & 100130155 & Hypothetical protein LOC100130155 \\
\hline 242246_x_at & 17.554796 & & & \\
\hline 242316_at & 27.534788 & & & \\
\hline 242509_at & 21.54953 & & & \\
\hline
\end{tabular}


Table III. Continued.

\begin{tabular}{|c|c|c|c|c|}
\hline Probe set ID & Fold change & Gene symbol & Entrez gene & Gene title \\
\hline 242906_at & 27.747772 & & & \\
\hline 242943_at & 14.356349 & ST8SIA4 & 7903 & ST8 $\alpha$-N-acetyl-neuraminide $\alpha$-2,8-sialyltransferase 4 \\
\hline 243489_at & 14.7732115 & & & \\
\hline 244009_at & 10.311149 & & & \\
\hline 244074_at & 12.198139 & LOC100129104 & 100129104 & Similar to hydroxyproline-rich glycoprotein VSP-3 \\
\hline 244112_x_at & 17.781418 & & & \\
\hline 1552440_at & 22.730635 & GPR182 & 11318 & G protein-coupled receptor 182 \\
\hline 1552463_at & 17.636255 & SERPINB11 & 89778 & $\begin{array}{l}\text { Serpin peptidase inhibitor, clade B (ovalbumin), member } 11 \\
\text { (gene/pseudogene) }\end{array}$ \\
\hline 1552584_at & 11.233118 & IL12RB1 & 3594 & Interleukin 12 receptor, $\beta 1$ \\
\hline 1553470_at & 12.427377 & DNAH17 & 8632 & Dynein, axonemal, heavy chain 17 \\
\hline 1553626_a_at & 15.016963 & C17orf57 & 124989 & Chromosome 17 open reading frame 57 \\
\hline 1553970_s_at & 10.47964 & CEL & 1056 & Carboxyl ester lipase (bile salt-stimulated lipase) \\
\hline 1554037_a_at & 11.989659 & ZBTB24 & 9841 & Zinc finger and BTB domain containing 24 \\
\hline 1554277_s_at & 10.488272 & FANCM & 57697 & Fanconi anemia, complementation group $\mathrm{M}$ \\
\hline 1555745_a_at & 11.363364 & LYZ & 4069 & Lysozyme (renal amyloidosis) \\
\hline 1554672_at & 19.961636 & TTC26 & 79989 & Tetratricopeptide repeat domain 26 \\
\hline 1555002_at & 22.022196 & MGC39545 & 403312 & Hypothetical protein LOC403312 \\
\hline 1555022_at & 25.609352 & RGS12 & 6002 & Regulator of G-protein signaling 12 \\
\hline 1555339_at & 12.859835 & RAP1A & 5906 & RAP1A, member of RAS oncogene family \\
\hline 1555435_at & 36.40531 & AFF4 & 27125 & AF4/FMR2 family, member 4 \\
\hline 1556474_a_at & 52.13133 & FLJ38379 & 285097 & Hypothetical FLJ38379 \\
\hline 1556877_at & 25.908909 & & & \\
\hline 1556898_at & 11.959699 & LOC200830 & 200830 & Hypothetical protein LOC200830 \\
\hline 1556905_at & 32.662815 & ZNF577 & 84765 & Zinc finger protein 577 \\
\hline 1557581_x_at & 10.408871 & & & \\
\hline 1557753_at & 14.301804 & & & \\
\hline 1558452_at & 10.139205 & TMEM144 & 55314 & Transmembrane protein 144 \\
\hline 1558706_a_at & 18.524925 & ATOH8 & 84913 & Atonal homolog 8 (Drosophila) \\
\hline 1558999_x_at & 17.685259 & LOC283922 & 283922 & Pyruvate dehydrogenase phosphatase regulatory subunit pseudogene \\
\hline & & PDPR & 55066 & Pyruvate dehydrogenase phosphatase regulatory subunit \\
\hline 1559057_at & 13.380709 & & & \\
\hline 1559101_at & 11.838457 & FYN & 2534 & FYN oncogene related to SRC, FGR, YES \\
\hline 1560756_at & 22.905785 & & & \\
\hline 1560996_at & 22.832338 & & & \\
\hline 1560999_a_at & 38.31254 & & & \\
\hline 1561521_at & 27.853552 & S100B & 6285 & S100 calcium binding protein B \\
\hline 1561882_at & 37.053608 & SYTL3 & 94120 & Synaptotagmin-like 3 \\
\hline 1562094_at & 12.56786 & & & \\
\hline 1562165_at & 10.340052 & & & \\
\hline 1562367_at & 17.341137 & $\mathrm{C} 15$ or $\mathrm{f} 54$ & 400360 & Chromosome 15 open reading frame 54 \\
\hline 1562528_at & 24.569925 & & & \\
\hline 1562716_at & 11.445873 & LOC284632 & 284632 & Hypothetical LOC284632 \\
\hline 1562990_at & 15.226481 & & & \\
\hline 1563061_at & 30.11877 & & & \\
\hline 1563077_at & 14.259786 & & & \\
\hline 1563612_at & 25.13671 & & & \\
\hline 1563827_at & 13.029244 & LOC158434 & 158434 & Hypothetical protein LOC158434 \\
\hline 1564003_at & 17.397495 & & & \\
\hline 1564294_at & 12.534436 & & & \\
\hline 1564299_at & 25.326767 & LOC100127940 & 100127940 & Hypothetical LOC100127940 \\
\hline 1564386_at & 36.96616 & TXNDC8 & 255220 & Thioredoxin domain containing 8 (spermatozoa) \\
\hline 1564653_s_at & 17.913086 & LEKR1 & 389170 & Leucine, glutamate and lysine rich 1 \\
\hline 1565668_at & 15.500862 & TUBA1B & 10376 & Tubulin, $\alpha 1 \mathrm{~b}$ \\
\hline 1565755_at & 22.79346 & MAP1D & 254042 & Methionine aminopeptidase 1D \\
\hline 1566767_at & 12.185921 & & & \\
\hline 1567702_at & 19.35184 & & & \\
\hline 1568696_at & 24.071138 & ARMETL1 & 441549 & Arginine-rich, mutated in early stage tumors-like 1 \\
\hline 1569218_at & 40.51703 & & & \\
\hline 1569408_at & 26.201654 & EIF2C4 & 192670 & Eukaryotic translation initiation factor $2 \mathrm{C}, 4$ \\
\hline 1569788_at & 15.160305 & ST8SIA1 & 6489 & ST8 $\alpha$-N-acetyl-neuraminide $\alpha$-2,8-sialyltransferase 1 \\
\hline 1570486_at & 12.096198 & SOX6 & 55553 & SRY (sex determining region Y)-box 6 \\
\hline
\end{tabular}


colon carcinoma, in order to identify specific functional genes related to lymphatic and hepatic metastases.

\section{Acknowledgements}

The present study was supported by the State Key Basic Research and Development Program of China (973 Program, Grant No. 2009CB521704), the National High-tech Research and Development Program of China (863 Program, Grant No. 2006AA02A245), the National Natural Science Foundation of China (Grant No. 81000894), the Zhejiang Provincial Science and Technology Projects (Grant No. 2009C13021, 2011C23087), the Science Research Fund of Shaoxing (Grant No. 2011D10013) and the Science Research Fund of Zhuji (Grant No. 2011CC7874).

\section{References}

1. Jin KT, He KF, Teng F, Han N, Li GL, Xu ZZ and Teng LS: Heterogeneity in primary tumors and corresponding metastases: could it provide us with any hints to personalize cancer therapy? Pers Med 8: 175-182, 2011.

2. Gong Y, Booser DJ and Sneige N: Comparison of HER-2 status determined by fluorescence in situ hybridization in primary and metastatic breast carcinoma. Cancer 103: 1763-1769, 2005.

3. Gancberg D, Di Leo A, Cardoso F, Rouas G, Pedrocchi M, Paesmans M, Verhest A, Bernard-Marty C, Piccart MJ and Larsimont D: Comparison of HER-2 status between primary breast cancer and corresponding distant metastatic sites. Ann Oncol 13: 1036-1043, 2002.

4. Regitnig P, Schippinger W, Lindbauer M, Samonigg H and Lax SF: Change of HER-2/neu status in a subset of distant metastases from breast carcinomas. J Pathol 203: 918-926, 2004.

5. Bozzetti C, Personeni N, Nizzoli R, Guazzi A, Flora M, Bassano C, Negri F, Martella E, Naldi N, Franciosi V and Cascinu S: HER-2/neu amplification by fluorescence in situ hybridization in cytologic samples from distant metastatic sites of breast carcinoma. Cancer 99: 310-315, 2003.

6. Tanner M, Järvinen P and Isola J: Amplification of HER-2/neu and topoisomerase IIalpha in primary and metastatic breast cancer. Cancer Res 61: 5345-5348, 2001.

7. Tapia C, Savic S, Wagner U, Schönegg R, Novotny H, Grilli B, Herzog M, Barascud AD, Zlobec I, Cathomas G, Terracciano L, Feichter $\mathrm{G}$ and Bubendorf L: HER2 gene status in primary breast cancers and matched distant metastases. Breast Cancer Res 9: R31, 2007.

8. Akcakanat A, Sahin A, Shaye AN, Velasco MA and Meric-Bernstam F: Comparison of Akt/mTOR signaling in primary breast tumors and matched distant metastases. Cancer 112: 2352-2358, 2008.
9. Wu JM, Fackler MJ, Halushka MK, Molavi DW, Taylor ME, Teo WW, Griffin C, Fetting J, Davidson NE, De Marzo AM, Hicks JL, Chitale D, Ladanyi M, Sukumar S and Argani P: Heterogeneity of breast cancer metastases: comparison of therapeutic target expression and promoter methylation between primary tumors and their multifocal metastases. Clin Cancer Res 14: 1938-1946, 2008.

10. Baldus SE, Schaefer KL, Engers R, Hartleb D, Stoecklein NH and Gabbert HE: Prevalence and heterogeneity of KRAS, BRAF, and PIK3CA mutations in primary colorectal adenocarcinomas and their corresponding metastases. Clin Cancer Res 16: 790-799, 2010.

11. Molinari F, Martin V, Saletti P, De Dosso S, Spitale A, Camponovo A, Bordoni A, Crippa S, Mazzucchelli L and Frattini M: Differing deregulation of EGFR and downstream proteins in primary colorectal cancer and related metastatic sites may be clinically relevant. Br J Cancer 100: 1087-1094, 2009.

12. Scartozzi M, Bearzi I, Berardi R, Mandolesi A, Fabris G and Cascinu S: Epidermal growth factor receptor (EGFR) status in primary colorectal tumors does not correlate with EGFR expression in related metastatic sites: implications for treatment with EGFR-targeted monoclonal antibodies. J Clin Oncol 22: 4772-4778, 2004.

13. Scartozzi M, Bearzi I, Berardi R, Mandolesi A, Pierantoni C and Cascinu S: Epidermal growth factor receptor (EGFR) downstream signalling pathway in primary colorectal tumours and related metastatic sites: optimising EGFR-targeted treatment options. Br J Cancer 97: 92-97, 2007.

14. Sasatomi E, Finkelstein SD, Woods JD, Bakker A, Swalsky PA, Luketich JD, Fernando HC and Yousem SA: Comparison of accumulated allele loss between primary tumor and lymph node metastasis in stage II non-small cell lung carcinoma: implications for the timing of lymph node metastasis and prognostic value. Cancer Res 62: 2681-2689, 2002.

15. Park S, Holmes-Tisch AJ, Cho EY, Shim YM, Kim J, Kim HS, Lee J, Park YH, Ahn JS, Park K, Jänne PA and Ahn MJ: Discordance of molecular biomarkers associated with epidermal growth factor receptor pathway between primary tumors and lymph node metastasis in non-small cell lung cancer. J Thorac Oncol 4: 809-815, 2009.

16. Jin K, Li G, Cui B, Zhang J, Lan H, Han N, Xie B, Cao F, He K, Wang H, Xu Z, Teng L and Zhu T: Assessment of a novel VEGF targeted agent using patient-derived tumor tissue xenograft models of colon carcinoma with lymphatic and hepatic metastases. PLoS One 6: e28384, 2011.

17. Fichtner I, Rolff J, Soong R, Hoffmann J, Hammer S, Sommer A, Becker M and Merk J: Establishment of patient-derived non-small cell lung cancer xenografts as models for the identification of predictive biomarkers. Clin Cancer Res 14: 6456-6468, 2008

18. Jin KT, He KF, Li GL and Teng LS: Personalized cancer therapy using a patient-derived tumor tissue xenograft model: a translational field worthy of exploring further? Pers Med 7: 597-606, 2010 . 